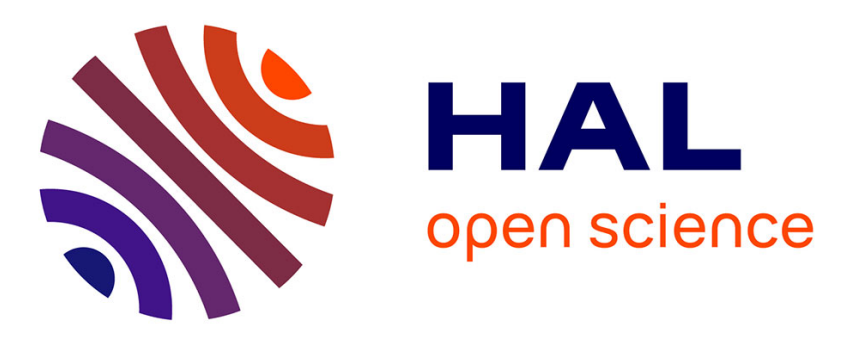

\title{
Morphological optimization of prosthesis' finger for precision grasping
}

\author{
J. Ramirez, A. Rubiano, N. Jouandeau, L. Gallimard, O. Polit
}

\section{To cite this version:}

J. Ramirez, A. Rubiano, N. Jouandeau, L. Gallimard, O. Polit. Morphological optimization of prosthesis' finger for precision grasping. 4th International workshop in medical and service robots, Jul 2015, Nantes, France. 10.1007/978-3-319-30674-2_19 . hal-02317209

\section{HAL Id: hal-02317209 \\ https://hal.science/hal-02317209}

Submitted on 15 Oct 2019

HAL is a multi-disciplinary open access archive for the deposit and dissemination of scientific research documents, whether they are published or not. The documents may come from teaching and research institutions in France or abroad, or from public or private research centers.
L'archive ouverte pluridisciplinaire HAL, est destinée au dépôt et à la diffusion de documents scientifiques de niveau recherche, publiés ou non, émanant des établissements d'enseignement et de recherche français ou étrangers, des laboratoires publics ou privés. 


\title{
Morphological optimization of prosthesis' finger for precision grasping
}

\author{
J. L. Ramírez ${ }^{1}$, A. Rubiano ${ }^{13}$, N. Jouandeau ${ }^{2}$, L. Gallimard ${ }^{1}$, O. Polit ${ }^{1}$ \\ ${ }^{1}$ LEME Université Paris Ouest Nanterre La Défense, France, \\ e-mail: jl.ramirez_arias@u-paris10.fr \\ ${ }^{2}$ LIASD Université Paris 8, France, e-mail: n@ai.univ-paris8.fr \\ ${ }^{3}$ Universidad Militar Nueva Granada, Colombia, \\ e-mail: astrid.rubiano@unimilitar.edu.co
}

\begin{abstract}
In this paper, we present the morphological optimization of our tendon driven underactuated robotic hand prosthesis' finger, to improve precision grasping. The optimization process is performed with a black box optimizer that considers simultaneously kinematic and dynamic constraints. The kinematic is computed with the Denhavit-Hartenberg parameterization modified by Khalil and Kleinfinger and the dynamic is computed from the virtual displacements and the virtual works. All these constraints are considered as a fitness function to evaluate the best morphological configuration of the finger. This approach gives a way to introduce and improve soft and flexible considerations for the grasping robots such as hands and grippers. Theoretical and experimental results show that flexible links combined with morphological optimization, lead in more precise grasping. The results of the optimization, show us an important improvement related to size, torque and consequently energy consumption.
\end{abstract}

Key words: Morphological optimization, mechanisms prehension, precision grasping, soft robotic

\section{Introduction}

Frequently, robots are designed as rigid structures, but recently some works have shown that the use of flexible bodies, actuators, and sensors could improve considerably the performance of the robots to interact with the environment, this kind of robots is known as soft robots. Soft robotic [1] systems could be able to improve grasping and manipulation, because they use: (i) elastic and deformable bodies, (ii) unconventional materials (like smart materials, shape memory alloy [2], i.a.) and (iii) high number of degrees of freedom.

In this context, the University of Bologna developed the UB-HAND IV, also called DEXMART Hand [3]. The hand is based on an endoskeletal structure articulated via pin joints, the actuators are located remotely with tendon-based transmissions, it has a soft cover and the mechanical structure of the hand was manufactured using additive technologies [4].

With the aim of building a robust and safe hand the University of Pisa and the Advanced Robotics Department of the Italian Institute of Technology in Genoa pro- 
posed the Pisa-IIT Soft Hand. This hand incorporated traditional and rolling contact joints with elastic ligaments. The rolling contact joints ensure a correct motion while the hand is actuated. In case of impacts, these joints provide an easy disengage and allow deformations. The hand has a single tendon, passing through all the joints simultaneously. The tendon produces flexion-extension and adduction-abduction of the fingers during the actuation [5].

Consequently, an important tool named Mooveit! [6] incorporates the latest advances in motion planning, manipulation, control and navigation of arms, grippers and hands i.a. Even if the tool is designed for rigid robots it constitutes an interesting approach.

The consideration of a tendon driven under-actuated mechanisms, flexible links and soft articulations in kinematics chains, introduces the necessity of optimal analysis of the morphological parameters. In this domain, one important development is presented by Jouandeau [7], which is related to "Enhancing Humanoids Walking Skills through Morphogenesis Evolution Method", in that work the optimization was performed using confident local optimization for noisy black-box parameter tuning (CLOP)[8].

Based on our first prototype, we observe that an optimization of the finger lengths is wide important to increase the precision and reduce the torque requirements. Consequently, considering that the target of our robotic hand is the precision grasping, we begin the design process with the optimization of the finger that is the key element of the robotic hand.

Therefore, in this paper we propose the application of CLOP to improve the performance of our tendon driven finger, during the precision grasping. Our approach is to use the mathematical model of the kinematic (based on the Denhavit-Hartenberg parameterization modified by Khalil and Kleinfinger) and dynamics (based on the virtual displacements and virtual works) as the fitness function to evaluate the best morphological configuration of the finger. This approach gives a way to introduce and improve soft and flexible considerations for the grasping robots such as hands and grippers.

\section{Kinematic and dynamic modelling of the robotic hand prosthesis' finger}

\subsection{Description of the robotic hand prosthesis' finger}

Our approach is a bio-inspired tendon-driven finger composed of three joints, the metacarpophalangeal (MP), the proximal interphalangeal (PIP) and the distal interphalangeal (DIP). The MP joint has two degrees of freedom (DoF); one rotation (to perform flexion-extension), and one passive translation that allows the vertical alignment of the joint with the motor axis. The PIP and DIP joints have one DoF to perform flexion and extension. 
The finger is under-actuated, so it is controlled by only one servo motor. The drive mechanism uses two tendons for transmitting motion to the finger, one tendon flex the finger and the other extend the finger. Considering that the tendons are attached to the motor pulley and the fingertip, as shown in Fig. 1, the clockwise rotation of the servo motor causes a flexion, and the anticlockwise rotation produce an extension. Due to the under actuation the rotation angles of the PIP $\left(\theta_{35}\right)$ and DIP $\left(\theta_{36}\right)$ joints are linked with the rotation angle of MP $\left(\theta_{33}\right)$ joint. The relation between angle is given as $\theta_{35}=0.23 \theta_{33}$ and $\theta_{36}=0.72 \theta_{33}$, where $\theta_{33}$ is the MP joint angle, $\theta_{35}$ is the PIP joint angle and $\theta_{36}$ is the DIP joint angle.
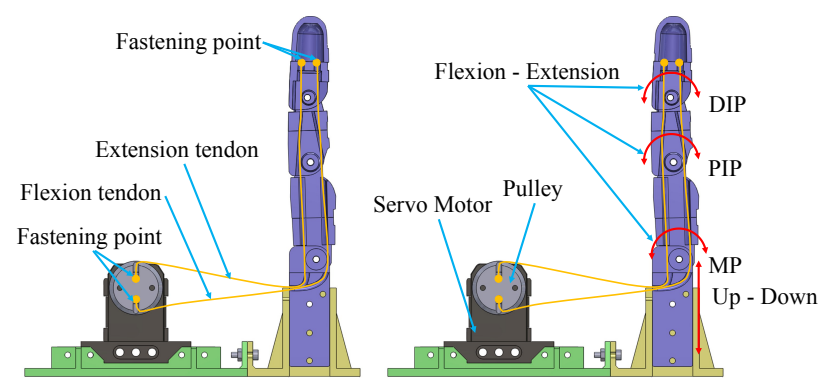

Fig. 1: Section view of the finger mechanism

The kinematic and the dynamic modeling are presented in the following subsections. For the kinematics, the Denhavit-Hartenberg parameterization modified by Khalil and Kleinfinger (DHKK) is used and for the dynamics we use the virtual displacements and virtual works approach. The equivalent mechanical model of the finger is shown in Fig. 2a for the kinematic and in Fig. $2 b$ for the dynamic.

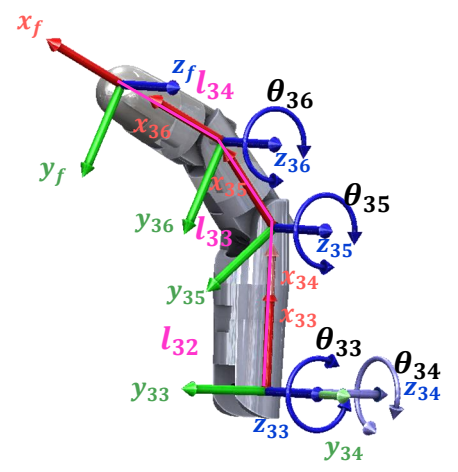

(a)

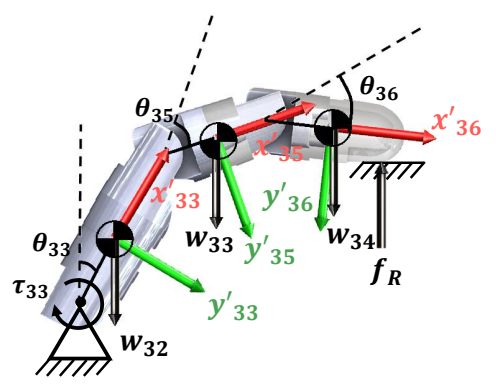

(b)

Fig. 2: Equivalent models of the finger. (a) Kinematic Model. (b) Dynamic model 
In Fig. 2a, the parameters $l_{32}, l_{33}$ and $l_{34}$ are the lengths of the proximal, medial and distal phalanges respectively. The angles $\theta_{33}, \theta_{34}, \theta_{35}$ and $\theta_{36}$ correspond to MP, PIP and DIP joints rotations. The frameworks $\left(x_{33}, y_{33}, z_{33}\right)$ and $\left(x_{34}, y_{34}, z_{34}\right)$ are associated to the MP joint. The framework $\left(x_{35}, y_{35}, z_{35}\right)$ is associated to the PIP joint and the framework $\left(x_{36}, y_{36}, z_{36}\right)$ associated to the DIP joint. The framework $\left(x_{f}, y_{f}, z_{f}\right)$ corresponds to the fingertip position. In the same way, in Fig. 2b, $w_{32}, w_{33}$ and $w_{34}$ are the weights of the proximal, medial and distal phalanges respectively, and are placed in the points $\left(x_{33}^{\prime}, y_{33}^{\prime}\right),\left(x_{35}^{\prime}, y_{35}^{\prime}\right)$ and $\left(x_{36}^{\prime}, y_{36}^{\prime}\right) . F_{r}$ is the applied force that is equivalent to the reaction force.

\subsection{Kinematic model of the robotic hand prosthesis' finger}

To propose a method to model the kinematic of our robotic hand prosthesis' finger, we use the DHKK. This convention, allows the representation of open-loop and close-loop kinematic chains, and presents a convenient definition of:

- The rotation axis $\mathbf{z}_{i}$ of each $i-t h$ joint

- The angle of rotation $\theta_{i}$ around $\mathbf{z}_{i}$

- The rotation $\alpha_{i}$ around $\mathbf{x}_{i-1}$

- The distance $a_{i}$ along of $\mathbf{x}_{i-1}$

- The distance $d_{i}$ along of $\mathbf{z}_{i}$

These parameters $\theta_{i}, \alpha_{i}, a_{i}$ and $d_{i}$ are known as DHKK parameters, and are calculated for each joint $M_{i}$ with coordinates $\left(x_{i}, y_{i}, z_{i}\right)$, in Fig. 3 a graphical representation of these DHKK parameters is presented. The rotations are performed using the transformation matrix shown in Eq. (1)[9].

$$
{ }^{i-1} T_{i}=\left[\begin{array}{cccc}
\cos \theta_{i} & \sin \theta_{i} & 0 & a_{i} \\
\sin \theta_{i} \cos \alpha_{i} & \cos \theta_{i} \cos \alpha_{i} & \sin \alpha_{i} & \sin \alpha_{i} d_{i} \\
\sin \theta_{i} \sin \alpha_{i} & \cos \theta_{i} \sin \alpha_{i} & \cos \alpha_{i} & \cos \alpha_{i} d_{i} \\
0 & 0 & 0 & 1
\end{array}\right]
$$

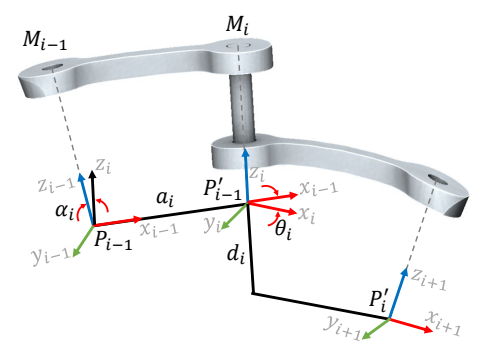

Fig. 3: Graphical representation of DHKK parameters 
Consequently, the kinematics of a robot composed of $n$ joints is the matrix ${ }^{0} T_{n}$, which is a composition of the $3 \times 3$ orientation matrix ${ }_{n}^{0} R$, and the position vector $\left[{ }^{0} P_{n}^{x},{ }^{0} P_{n}^{y},{ }^{0} P_{n}^{z}\right]^{T}$, as shown in the following expression:

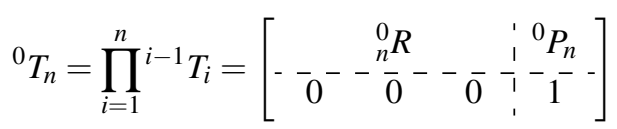

Usually, the formulation of DHKK parameters is difficult, because is performed manually, and is more difficult when there are multiple kinematic chains. Even that, a method to automatically generate these parameters is presented in [10], and is the adopted methodology to parameterize in the present work. The DHKK parameter for our finger are shown in Table 1, using the configuration shown in Fig. 2a.

Table 1: DHKK Parameters of the robotic hand prosthesis' finger.

\begin{tabular}{lllll}
\hline Link & $\alpha_{i}$ & $a_{i}$ & $d_{i}$ & $\theta_{i}$ \\
\hline 33 & $-\pi / 2$ & 0 & 0 & $\theta_{33}$ \\
34 & $\pi / 2$ & 0 & 0 & $\theta_{34}$ \\
35 & $-\pi / 2$ & $l_{32}$ & 0 & $\theta_{35}$ \\
36 & 0 & $l_{33}$ & 0 & $\theta_{36}$ \\
$f$ & 0 & $l_{34}$ & 0 & 0 \\
\hline
\end{tabular}

Operating the Eq. (1) with the DHKK parameters of the robotic hand prosthesis' finger, shown in Table 1 , we get a set of five matrices ${ }^{0} T_{33},{ }^{33} T_{34},{ }^{34} T_{35}$, ${ }^{35} T_{36}$ and ${ }^{36} T_{f}$, that describe the transformation between the links of the kinematic chain. Applying the Eq. (2) with the calculated ${ }^{i-1} T_{i}$ matrices, we get the position $\left[{ }^{0} P_{n}^{x},{ }^{0} P_{n}^{y},{ }^{0} P_{n}^{z}\right]^{T}$ and orientation ${ }_{n}^{0} R$ of the joints.

Considering that the frameworks $M_{33}$ and $M_{34}$ are in the same position of the reference framework, the position vectors of ${ }^{0} T_{33}$ and ${ }^{0} T_{34}$ are zero (see Eq. (3), and Eq. (4)). From Eq. (3) to Eq. (7) we use the abbreviations $C_{i}:=\cos \left(\theta_{i}\right)$ and $S_{i}:=\sin \left(\theta_{i}\right)$.

$$
\begin{gathered}
{ }^{0} T_{33}=\left[\begin{array}{ccc:c}
C_{33} & -S_{33} & 0 & 0 \\
0 & 0 & 1 & 0 \\
-S_{33}-C_{33} & 0 & 0 \\
\hdashline 0 & 0 & 0 & 1
\end{array}\right] \\
{ }^{0} T_{34}=\left[\begin{array}{ccc:c}
C_{33} C_{34} & -C_{33} S_{34} & S_{33} & 0 \\
S_{34} & C_{34} & 0 & 0 \\
-C_{34} S_{33} & S_{33} S_{34} & C_{33} & 0 \\
\hdashline 0 & 0 & 0 & 1
\end{array}\right]
\end{gathered}
$$

The following equation corresponds to the position $\left[{ }^{0} P_{35}^{x},{ }^{0} P_{35}^{y},{ }^{0} P_{35}^{z}\right]^{T}$ and orientation ${ }_{35}^{0} R$ of the joint $M_{35}$. 


$$
{ }^{0} T_{35}=\left[\begin{array}{ccc:c}
C_{33} C_{34} C_{35}-S_{33} S_{35} & -C_{35} S_{33}-C_{33} C_{34} S_{35} & -C_{33} S_{34} & l_{32} C_{33} C_{34} \\
C_{35} S_{34} & -S_{34} S_{35} & C_{34} & l_{32} S_{34} \\
-C_{33} S_{35}-C_{34} C_{35} S_{33} & C_{34} S_{33} S_{35}-C_{33} C_{35} & S_{33} S_{34} & -l_{32} C_{34} S_{33} \\
\hdashline 0 & 0 & 0 & 1
\end{array}\right]
$$

The following equation corresponds to the position $\left[{ }^{0} P_{36}^{x},{ }^{0} P_{36}^{y},{ }^{0} P_{36}^{z}\right]^{T}$ and orientation ${ }_{36}^{0} R$ of the joint $M_{36}$.

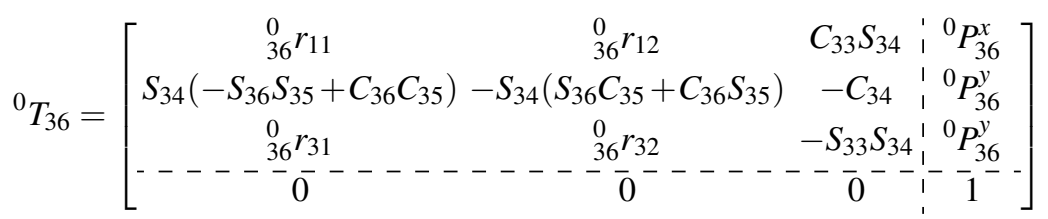

$$
\begin{aligned}
& \text { where }{ }_{36}^{0} r_{11}=C_{34}\left(-S_{36} S_{35}+C_{36} C_{35}\right) C_{33}-S_{33}\left(S_{36} C_{35}+C_{36} S_{35}\right) \\
& { }_{36}^{0} r_{12}=-C_{34}\left(S_{36} C_{35}+C_{36} S_{35}\right) C_{33}-S_{33}\left(-S_{36} S_{35}+C_{36} C_{35}\right) \\
& { }_{36}^{0} r_{31}=\left(-S_{36} C_{35}-C_{36} S_{35}\right) C_{33}-C_{34} S_{33}\left(-S_{36} S_{35}+C_{36} C_{35}\right) \\
& { }_{36}^{0} r_{32}=\left(S_{36} S_{35}-C_{36} C_{35}\right) C_{33}+C_{34} S_{33}\left(S_{36} C_{35}+C_{36} S_{35}\right) \\
& { }^{0} P_{36}^{x}=C_{34}\left(l_{32}+l_{33} C_{35}\right) C_{33}-l_{33} S_{33} S_{35} \\
& { }^{0} P_{36}^{y}=S_{34}\left(l_{32}+l_{33} C_{35}\right) \\
& { }^{0} P_{36}^{z}=-S_{33}\left(l_{32}+l_{33} C_{35}\right) C_{34}-l_{33} C_{33} S_{35}
\end{aligned}
$$

The following equation corresponds to the position $\left[{ }^{0} P_{f}^{x},{ }^{0} P_{f}^{y},{ }^{0} P_{f}^{z}\right]^{T}$ and orientation ${ }_{f}^{0} R$ of the fingertip.

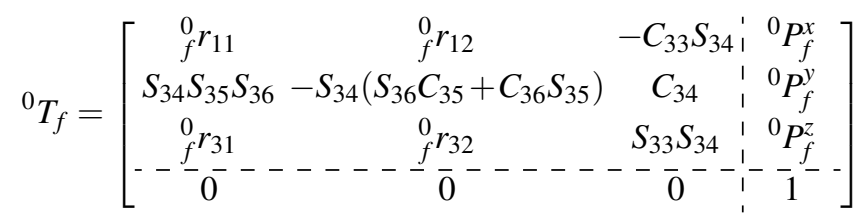

where ${ }_{f}^{0} r_{11}=C_{34}\left(-S_{36} S_{35}+C_{36} C_{35}\right) C_{33}-S_{33}\left(S_{36} C_{35}+C_{36} S_{35}\right)$

$$
\begin{aligned}
{ }_{f}^{0} r_{12}= & -C_{34}\left(S_{36} C_{35}+C_{36} S_{35}\right) C_{33}-S_{33}\left(-S_{36} S_{35}+C_{36} C_{35}\right) \\
{ }_{0}^{0} r_{31}= & \left(-S_{36} C_{35}-C_{36} S_{35}\right) C_{33}-C_{34} S_{33}\left(-S_{36} S_{35}+C_{36} C_{35}\right) \\
{ }^{0} r_{32}= & \left(S_{36} S_{35}-C_{36} C_{35}\right) C_{33}+C_{34} S_{33}\left(S_{36} C_{35}+C_{36} S_{35}\right) \\
{ }^{0} P_{f}^{x}= & \left(\left(l_{33}+l_{34} C_{36}\right) C_{35}+l_{32}-l_{34} S_{36} S_{35}\right) C_{34} C_{33} \\
& -\left(C_{35} S_{36} l_{34}+S_{35}\left(l_{33}+l_{34} C_{36}\right)\right) S_{33} \\
{ }^{0} P_{f}^{y}= & \left(\left(l_{33}+l_{34} C_{36}\right) C_{35}+l_{32}-l_{34} S_{36} S_{35}\right) S_{34} \\
{ }^{0} P_{f}^{z}= & -\left(\left(l_{33}+l_{34} C_{36}\right) C_{35}+l_{32}-l_{34} S_{36} S_{35}\right) S_{33} C_{34} \\
& -C_{33}\left(C_{35} S_{36} l_{34}+S_{35}\left(l_{33}+l_{34} C_{36}\right)\right)
\end{aligned}
$$




\subsection{Dynamic model of the robotic hand prosthesis' finger}

The proposed dynamic model uses the principle of the virtual displacements and virtual works [11]. We use the dynamic equilibrium to define a model of the input torque as function of the applied force. The virtual work $\delta W$ is calculated for the external forces (e.g. weight, applied force and input torque) in Eq. (8) and the inertial forces (e.g. centrifugal forces) in Eq. (9).

$$
\delta W_{e}=Q_{e}^{T} \delta r_{e}
$$

where $Q_{e}^{T}$ is the external forces vector and $\delta r_{e}$ is the virtual displacement vector of the forces application points.

$$
\delta W_{i}=M \ddot{q}^{T} \delta r_{i}
$$

where $M$ is the diagonal mass matrix composed of the masses $m_{i}$ and inertias $J_{i}, \ddot{q}^{T}$ is the acceleration vector and $\delta r_{i}$ is the virtual displacement vector of the inertial frameworks.

The dynamic equilibrium is given by Eq. (10), but as in our model the rigid bodies have movements restrictions, the displacements in the points where forces are applied aren't independent, so to solve the equilibrium equation it is necessary separate the coordinates into dependent and independent coordinates.

$$
\delta q^{T}\left[M \ddot{q}-Q_{e}\right]=0
$$

In order to separate coordinates, the transformation shown in Eq. (11) is proposed, as result we have the equilibrium equation proposed in Eq. (12), which is separable.

$$
\delta q=B \delta q_{i i}, B=\left[\begin{array}{c}
-C_{q d}^{-1} C_{q i} \\
I
\end{array}\right]
$$

where $C_{q d}$ is the jacobian of dependent coordinates, $C_{q i}$ is the jacobian of independent coordinates and $I$ is the identity matrix.

$$
\delta q_{i i}^{T} B^{T}\left[M \ddot{q}-Q_{e}\right]=0
$$

Solving the equation Eq. (12), we obtain the dynamic function which give us the input torque $\tau_{33}$ as function of the force $\mathrm{Fr}$ and the kinematics $q, \dot{q}, \ddot{q}$. The resulting expression is shown in Eq. (13), where we use the abbreviations $C_{i}:=\cos \left(\theta_{i}\right)$ and $S_{i}:=\sin \left(\theta_{i}\right)$.

$$
\tau_{33}(F r, q, \dot{q}, \ddot{q})=\frac{H_{0}-4 l_{32} H_{12}+H_{13}\left(l_{33} S_{35}-4\right)}{8 l_{33} S_{35}-32}
$$

where $H_{0}=2 l_{32}{ }^{2} \ddot{\theta}_{33}\left(l_{33}\left(m_{33}+m_{32}\right) S_{35}-6 m_{33}-4 m_{32}\right)\left(S_{33}\right)^{2}$

$$
\begin{aligned}
& H_{1}=\left(\left(3 \ddot{\theta}_{35}+\ddot{x}_{33}\right) m_{33}+2 \ddot{\theta}_{35} m_{34}+\left(m_{34}+2 m_{33}+m_{32}\right) g-2 F r\right) l_{33} S_{35} \\
& H_{2}=\left(\left(m_{33}+2 m_{34}\right) l_{33}{ }^{2}+4 m_{34} l_{33} l_{34}+2 m_{34} l_{34}{ }^{2}\right)
\end{aligned}
$$




$$
\begin{aligned}
& H_{3}=\left(\left(1 / 2 m_{33} \ddot{y}_{33}+m_{34} \ddot{y}_{35}\right) l_{33}+l_{34} \ddot{y}_{35} m_{34}\right) C_{35} \\
& H_{4}=2 \ddot{\theta}_{36} S_{36} l_{34} m_{34}-6 \ddot{x}_{33} m_{33}+2 J_{33} \ddot{\theta}_{35}-4 \ddot{x}_{35} m_{34} \\
& H_{5}=\left(-6 m_{33}-4 m_{32}-4 m_{34}\right) g \\
& H_{6}=1 / 4 l_{32}^{2} \ddot{\theta}_{33}\left(3 / 2 m_{33}+m_{32}\right)\left(C_{33}\right)^{2} \\
& H_{7}=\left(\left(m_{33}+2 / 3 m_{34}\right) l_{33}+2 / 3 l_{34} m_{34}\right) \ddot{\theta}_{35} C_{35} \\
& H_{11}=1 / 4 m_{33} l_{33} C_{3} 5 l_{32} C_{3} 3 \ddot{\theta}_{33}+1 / 4 \ddot{\theta}_{35} H_{2} C_{35}{ }^{2} \\
& H_{12}=\left(-1 / 2 m_{33} l_{33} S_{35} \ddot{\theta}_{35}+H_{1}+H_{11}+H_{3}+H_{4}+H_{5}+8 F r\right) S_{33} \\
& H_{13}=8\left(H_{6}+3 / 8 l_{32}\left(H_{7}+2 m_{33} \ddot{y}_{33}+4 / 3 m_{34} \ddot{y}_{35}\right) C_{3} 3+J_{32} \ddot{\theta}_{33}\right)
\end{aligned}
$$

\section{Finger prototype test platform set-up}

With the aim of test the drive mechanism, we design a platform to carry out several experiments, the experiments seek to measure the kinematics of the finger and fingertip force using several servo motors. The platform permits to adjust the position of the MP joint with respect to the actuator axis, which guarantees that the actuator torque is transmitted to the tendons in the same condition. The CAD model of the test platform is shown in Fig. 4.

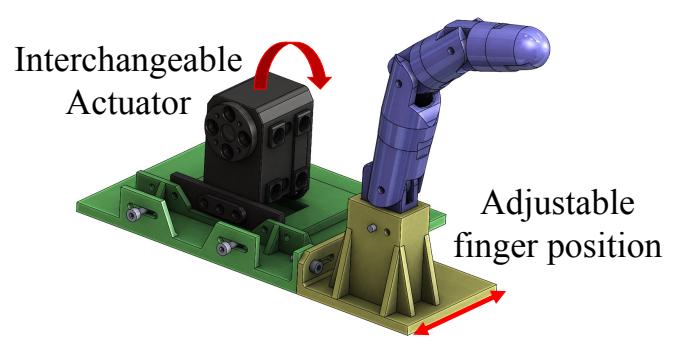

Fig. 4: CAD Model of the test platforms

\subsection{Materials and methods}

The experiments are performed using two standard servo motors HS-422 and Traxxas 2065 with torques of $0.324 \mathrm{Nm}$ and $0.225 \mathrm{Nm}$ respectively, and three serial servo motors Dynamixel XL-320, AX-12a and MX-106R with torques of $0.390 \mathrm{Nm}$, $1.50 \mathrm{Nm}$ and $10.0 \mathrm{Nm}$ respectively. To measure the force, we use a resistive-based force sensor Flexiforce $\AA$, that measure up to $5 N$, connected to a circuit that uses an inverting operational amplifier arrangement to produce an analog output based on the sensor resistance, the output voltage is registered with a digital oscilloscope. 
The sensor is calibrated in the range $0.6 \mathrm{~N}$ to $4.8 \mathrm{~N}$. The sensor is placed in a support (platform) which is located in the trajectory of the fingertip.

Considering that the finger performs flexion and extension in 2D, the kinematic is measured using a high-performance 4 megapixel CCD camera Prosilica GE-2040, to track black markers placed on the finger joints and the fingertip.

\subsection{Kinematic tracking and force measure}

As result of the test, shown in Fig. 5, which corresponds to the measure of the position of each $M_{i}$ joint (using the Traxxas 2065 servo motor), it is possible to identify several peaks, i.e. the ${ }^{0} P_{f}^{x}$ value shows a perturbation after contact. These overshoots are produced by different phenomena as friction forces and other external causes. The maximal force value is $2.82 N$, (using the Traxxas 2065 servo motor) Fig. 6 shows the fingertip force $f_{r}$ during three repetitions of flexion and extension.
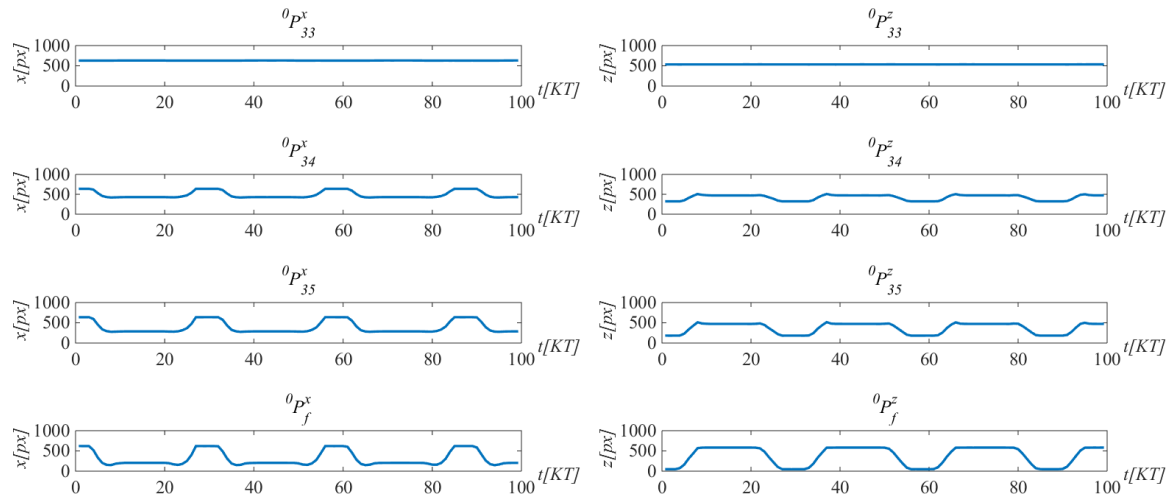

Fig. 5: Results of the position tracking using the Traxxas 2065 servo motor

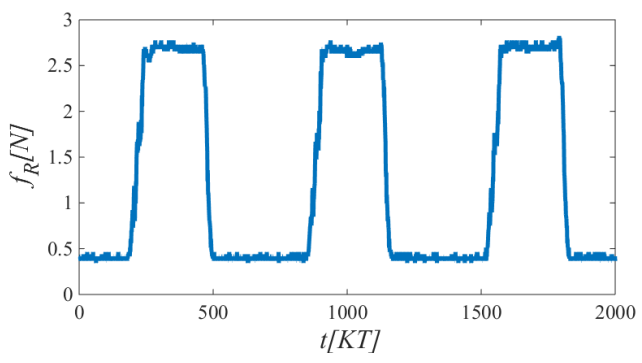

Fig. 6: Results of the fingertip force using the Traxxas 2065 servo motor 
Although we cannot control external conditions, we identify that the performance of the finger is affected by the phalanges lengths. The lengths of the finger could increase the amount of torque needed to apply the same force over an object, and also may impact the precision of the grasping. So that, we propose a morphological optimization of the lengths of the finger phalanges, which is presented in Section 4.

\section{Morphology optimization}

This section introduces the proposed optimization process, which improves the movement of the finger during the precision grasping. This process seeks to find the optimal lengths of the finger phalanges, to reduce the position error during a grasping movement and also to reduce the torque $\tau_{33}$ to apply a force of $5 \mathrm{~N}$.

The used method is confident local optimization (CLOP) for noisy black-box parameter tuning, which was developed to tune parameters in artificial intelligence for games. In our case, we follow the methodology proposed in [7] for enhancing the humanoids walking skills through morphogenesis evolution so that CLOP is implemented in two stages: evolution and evaluation.

\subsection{Evolution process}

The evolution process (based on an heuristic evaluation) uses as parameters the set $\mathscr{H}$ (which contains Motors, Torques $\langle M, T\rangle$ and length $s_{\text {new }}$ parameters, and their maximum and minimum values) and the eval function to calculate the fitness of the results. For each iteration, a new set of lengths is calculated and evaluated, the best result is updated when the founded solution is better than the stored one. The evolution process is presented in Alg. 1.

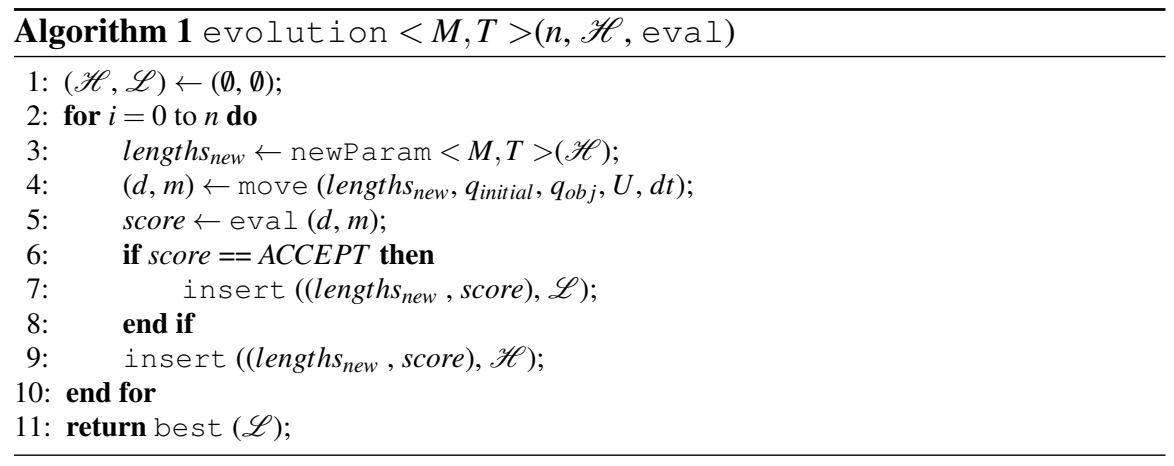




\subsection{Evaluation process}

The evaluation, use the results of the kinematic and the dynamic simulation to evaluate the fitness. The eval function determines two Euclidian distances: (i) distance $d$ between the fingertip and the objective position (positioning error), and (ii) distance $d_{\text {best }}$ between the best position founded and the objective position. Both distances are compared and the best result is updated only if $d$ is lower than $d_{\text {best }}$. In the same way, the function analyses if the input torque $m$ (which corresponds to $\tau_{33}$ ) is lower than the best to modify the result. The eval function is presented in Alg. 2

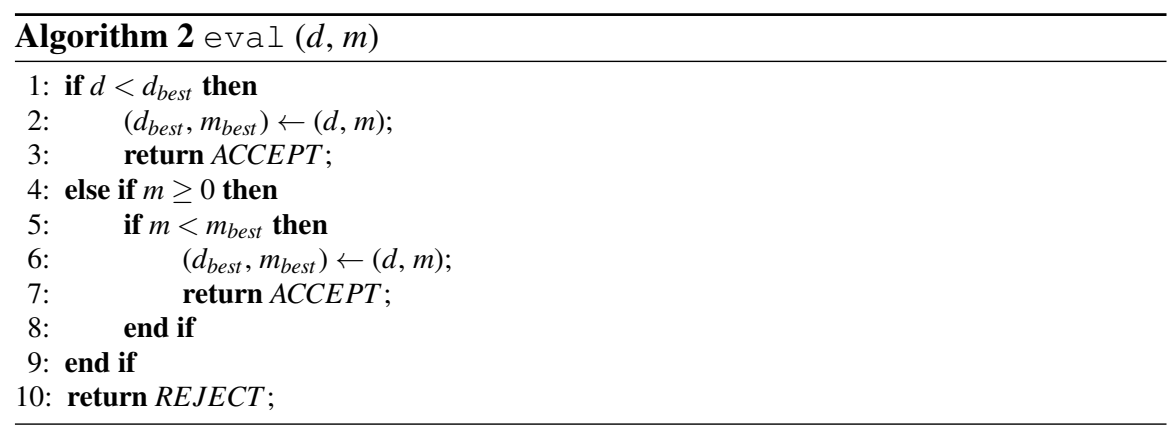

\subsection{Experiment setting and application}

To perform the experiment, two functions are defined, $f$ (lengths, $q, u, d t)$ to describe the movement before the contact with the object to grasp and $g($ lengths, $x, u, d t)$ to describe the movement after the contact with the object.

Before contact, the parameter $u$ is a speed control law defined as a ramp, which handle the engine speed to a maximal value with a constant slope. After contact $u$ is a force control law that handles the applied force to a maximal value $F r$ with a constant slope. The algorithms to implement both functions are presented in Alg. 3 and Alg. 4.

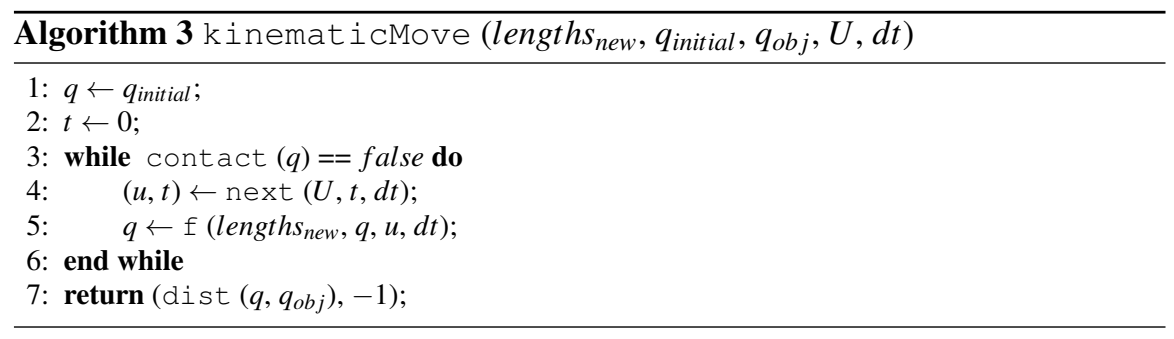




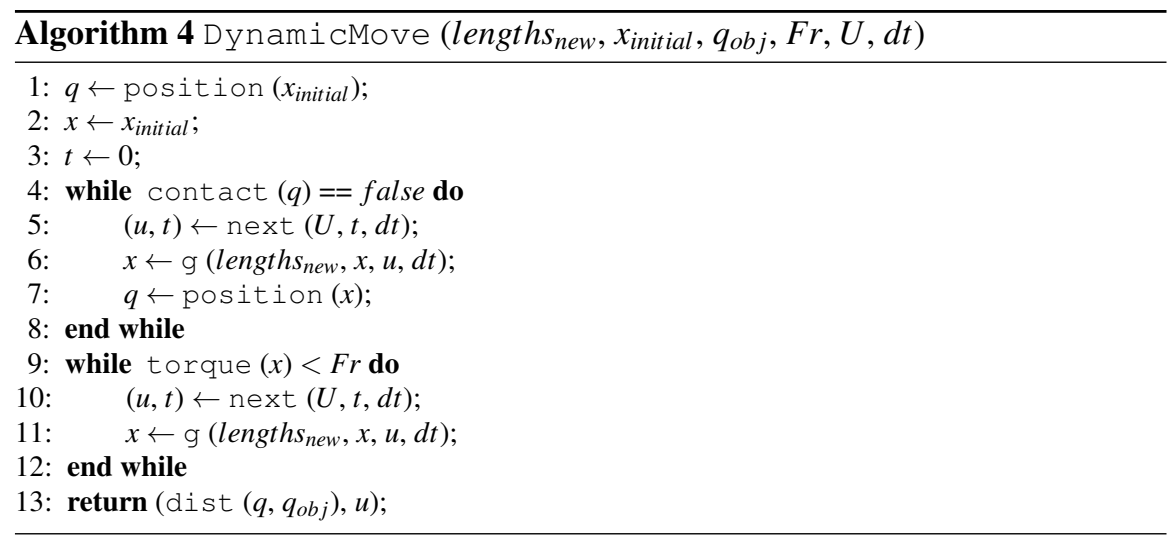

\section{Results}

The experiment, which was executed 150 times, search to minimize the position error and the torque $\tau_{33}$. The evaluation process generates iteratively solutions, from the first (where $l_{32}$ is $22.1 \mathrm{~mm}$ and $\tau_{33}$ is $46 \mathrm{Nmm}$ ) to the last (where $l_{32}$ is $22.2 \mathrm{~mm}$ and $\tau_{33}$ is $50.7 \mathrm{Nmm}$ ). Each solution is equivalent or better than the previous. By applying the morphological optimization on $l_{32}, l_{33}, l_{34}$ and $\tau_{33}$ parameters that produce $f_{R}$, we change parameters from $(36.6 \mathrm{~mm} 24 \mathrm{~mm} 25 \mathrm{~mm} 225 \mathrm{Nmm})$ to $(22.2 \mathrm{~mm}$ $9.2 \mathrm{~mm} 10 \mathrm{~mm} 50.7 \mathrm{Nmm}$ ) that improves $f_{R}$ by a factor of 2 with a single finger experiment. Such gain over output force on each finger will be more important with a prosthetic hand with multiple fingers.

Table 2: Seven best results of the CLOP experiments.

\begin{tabular}{llll}
\hline$l_{32}[\mathrm{~mm}]$ & $l_{33}[\mathrm{~mm}]$ & $l_{34}[\mathrm{~mm}]$ & $\tau_{33}[\mathrm{Nmm}]$ \\
\hline 22.1 & 19.6 & 16 & 46 \\
26.5 & 15.3 & 13.3 & 58.6 \\
26.2 & 10 & 18.9 & 55.1 \\
23.1 & 12.9 & 10.6 & 52.3 \\
22.9 & 12.8 & 10.3 & 51.8 \\
18.9 & 9.9 & 13.1 & 40.3 \\
22.2 & 9.2 & 10 & 50.7 \\
\hline
\end{tabular}

The complete experiment, corresponding to the last row of Table. 2, is shown in Fig. 7a, where is possible to follow the evolution of the phalanges lengths and the trajectory described from the initial position to the objective position. Consequently, the result shows a direct relationship between the length of the phalange $l_{32}$ and the 
torque $\tau_{33}$, and as can be shown in the Fig. $7 \mathrm{~b}$, the values are converging around a mean value.

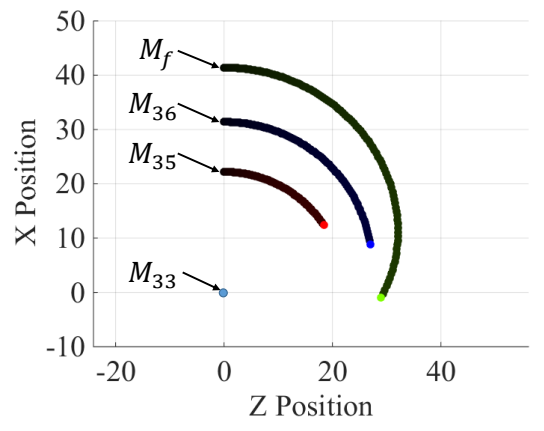

(a)

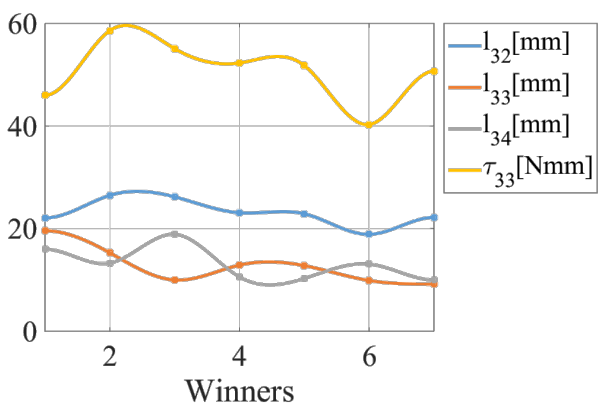

(b)

Fig. 7: Equivalent models of the finger. (a) Kinematic Model. (b) Dynamic model

\section{Perspectives}

The consideration of a tendon driven under-actuated mechanisms, introduces the necessity of torque control after the contact with the object to grasp, which in our case is the result of the optimal analysis of the morphological parameters. The morphological optimization performed, leads us to envisage a new finger, which should involve soft and flexible considerations for the design of the links and articulations, in order to achieve the grasping task in a more appropriate way. The results of the optimization, show us an important improvement related to size, torque and consequently energy consumption. Therefore, the next stage will be the integration of the optimal finger. Further investigations should also be tested to improve usability of robotic hand prosthesis with soft and flexible components.

\section{Conclusions}

The CLOP optimizer is a performant method that deals automatically with nonnegative Hessians, which is the case of the proposed dynamic method. Consequently, the morphological optimal values allow to propose an evolution of our robotic finger. The obtained results show a smooth trajectory that is important for precision grasping. A similar optimization process could be applied to create an adaptive torque control grasping system. 
The results of the performed torque optimization, give us an energetic performance, which render the robotic finger ideal to be used in our hand prosthesis. This energetic performance also lets the robotic hand prosthesis be more convenient to the patient according to the fact that we reduce the weight and increase the autonomy of the robot.

Acknowledgements Through this acknowledgement, we express our sincere gratitude to the Université Paris Lumières UPL for the financial support through the project PROMAIN This work has been partly supported by Université Paris Lumières UPL and by a Short Term Scientific Mission funding from LEME-UPO-EA4416 / LIASD-UP8-EA4383. We also acknowledge Colciencias Colombia and the Universidad Militar Nueva Granada for the financial support of the PhD students.

\section{References}

1. Nurzaman, S., Iida, F., Laschi, C., Ishiguro, A., Wood, R.: Soft robotics [tc spotlight]. Robotics Automation Magazine, IEEE 20(3), 24-95 (2013)

2. Andrianesis, K., Tzes, A.: Design of an innovative prosthetic hand with compact shape memory alloy actuators. In: 21st Mediterranean Conference on Control Automation (MED), pp. 697-702 (2013)

3. Palli, G., Scarcia, U., Melchiorri, C., Vassura, G.: Development of robotic hands: The ub hand evolution. In: IEEE/RSJ International Conference on Intelligent Robots and Systems (IROS), pp. 5456-5457 (2012)

4. Ficuciello, F., Palli, G., Melchiorri, C., Siciliano, B.: Postural synergies of the $\{U B\}$ hand $\{\mathrm{IV}\}$ for human-like grasping. Robotics and Autonomous Systems 62(4), 515 - 527 (2014)

5. Ajoudani, A., Godfrey, S., Catalano, M., Grioli, G., Tsagarakis, N., Bicchi, A.: Teleimpedance control of a synergy-driven anthropomorphic hand. In: IEEE/RSJ International Conference on Intelligent Robots and Systems (IROS), pp. 1985-1991 (2013)

6. Chitta, S., Sucan, I., Cousins, S.: Moveit! [ros topics]. IEEE Robotics Automation Magazine 19(1), 18-19 (2012)

7. Jouandeau, N., Hugel, V.: Enhancing humanoids walking skills through morphogenesis evolution method. In: D. Brugali, J. Broenink, T. Kroeger, B. MacDonald (eds.) Simulation, Modeling, and Programming for Autonomous Robots, Lecture Notes in Computer Science, vol. 8810, pp. 412-423. Springer International Publishing (2014)

8. Coulom, R.: Clop: Confident local optimization for noisyblack-box parameter tuning. In: H. van den Herik, A. Plaat (eds.) Advances in Computer Games, Lecture Notes in Computer Science, vol. 7168, pp. 146-157. Springer Berlin Heidelberg (2012)

9. Harada, K., Anzai, T.: Multiple sweeping using quaternion operations. Computer-Aided Design 34(11), 815-822 (2002)

10. Hugel, V., Jouandeau, N.: Automatic generation of humanoids geometric model parameters. In: RoboCup 2013: Robot World Cup XVII, pp. 408-419. Springer (2014)

11. Yamane, K., Nakamura, Y.: O(n) forward dynamics computation of open kinematic chains based on the principle of virtual work. In: IEEE International Conference on Robotics and AutomationICRA, vol. 3, pp. 2824-2831 (2001) 